\title{
RF energy harvesting: an analysis of wireless sensor networks for reliable communication
}

\author{
Hung $\operatorname{Tran}^{1} \cdot$ Johan Åkerberg ${ }^{2} \cdot$ Mats Björkman $^{1} \cdot$ Ha-Vu Tran ${ }^{3}$
}

Published online: 28 June 2017

(c) The Author(s) 2017. This article is an open access publication

\begin{abstract}
In this paper, we consider a wireless energy harvesting network consisting of one hybrid access point (HAP) having multiple antennas, and multiple sensor nodes each equipped with a single antenna. In contrast to conventional uplink wireless networks, the sensor nodes in the considered network have no embedded energy supply. They need to recharge the energy from the wireless signals broadcasted by the HAP in order to communicate. Based on the point-to-point and multipoints-to-point model, we propose two medium access control protocols, namely harvesting at the header of timeslot (HHT) and harvesting at the dedicated timeslot (HDT), in which the sensor nodes harvest energy from the HAP in the downlink, and then transform its stored packet into bit streams to send to the HAP in the uplink. Considering a deadline for each packet, the cumulative distribution functions of packet transmission time of the proposed protocols are derived for the selection combining and maximal ratio combining (MRC) techniques at the HAP. Subsequently, analytical expressions for the packet timeout probability and system
\end{abstract}

Hung Tran

tran.hung@mdh.se

Johan Åkerberg

johan.akerberg@mdh.se

Mats Björkman

mats.bjorkman@mdh.se

Ha-Vu Tran

ha-vu.tran.1@ens.etsmtl.ca

1 School of Innovation, Design and Engineering, Mälardalen University, Västerås, Sweden

$2 \mathrm{ABB} \mathrm{AB}$, Corporate Research, Västerås, Sweden

3 LACIME Laboratory, ETS Engineering School, University of Québec, Montreal, Canada reliability are obtained to analyze the performance of proposed protocols. Analytical results are validated by numerical simulations. The impacts of the system parameters, such as energy harvesting efficiency coefficient, sensor positions, transmit signal-to-noise ratio, and the length of energy harvesting time on the packet timeout probability and the system reliability are extensively investigated. Our results show that the performance of the HDT protocol outperforms the one using the HHT protocol, and the HDT protocol with the MRC technique has the best performance and it can be a potential solution to enhance the reliability for wireless sensor networks.

Keywords Energy harvesting - Wireless power transfer . Wireless sensor networks - Packet transmission time . Reliable communication

\section{Introduction}

Over the last few years, the industrial wireless sensor network (IWSN) has become one of the most interesting topics in the research community due to flexible installation and easy maintenance. Accordingly, many standards such as WirelessHART, WIA-PA, and ISA100.11a have been proposed [1-7]. More specifically, a dynamic power allocation policy for a wireless sensor network has been studied in [5] to improve the throughput and reduce energy consumption. In [6], authors investigated a strategy to set the time length in LEACH protocol to prolong the lifetime and increase throughput of wireless sensor network. In [7], an experiment study to understand the impact of interference among users on packet delivery ratio and throughput has been analyzed for wireless body sensor networks. Although, there are many works focusing on wireless 
sensor networks, but to fulfill demands on high reliability and timeliness is not easy because the wireless channels are often subject to interference and fading [3,8]. Additionally, as the size of sensor network increases, replacing or recharging the batteries takes time and costs. This work becomes dangerous for humans in hazardous environments such as nuclear reactors, toxic environments. Moreover, devices implant inside the human body are more difficult or impossible to replace. To overcome these drawbacks, the radio frequency (RF) energy harvesting for wireless sensor networks has been considered as a promising solution to prolong the sensor's lifetime and to enhance reliable communication $[9,10]$.

Recently, wireless power technologies have made a great progress to enable the wireless power transfer (WPT) for real wireless applications [11-14]. The wireless power can be harvested from natural sources such as solar, wind, TV broadcast signals, or a dedicated power transmitter [11]. In [12], a prototype of the RF energy harvesting device has been developed for experimental purposes. In $[15,16]$, authors have shown that the harvested energy can be stored in a supercapacitor, which can be charged very fast and the lifetime may be prolonged for many years with charging and discharging cycles. However, the power of the supercapacitor is often leaked out due to its self-discharge process, and it is not possible to store the harvested energy long enough for the next communication round. Without doubt, the applications of the RF energy harvesting will be used widely in near future, and this technology is still an open problem demanding more research.

In the light of RF energy harvesting ideas, many researchers have investigated on the problems of simultaneous information transmission and WPT in order to improve reliable communication, accordingly theoretical models, protocols, and system designs, have been proposed [17-28]. Specifically, in [17], an outage minimization with energy harvesting for point-to-point communication over fading channels has been studied. Employing a Markov model, the impact of packet retransmission, energy harvesting, and detection on the outage performance for a wireless power sensor network (WPSN) have been illustrated. Regarding to point-to-multipoint communications, Tiangquing et al. have focused on the problem of energy harvesting with cooperation beam selection for wireless sensors [18]. Closed-form expression for the distribution function of harvested energy in a coherent time is derived to analyze the system performance. In [20], the impact of energy harvesting on the packet loss probability and the average packet delay for overlaying wireless sensor networks has been considered. Also, the optimal design of energy storage capacity in the sensors has been proposed. Taking the advantages of cooperative communication, works reported in [26] have shown an interesting result that the harvested energy from a wireless source can obtain the same diversity multiplexing tradeoff as if the relay is attached to a fixed power supply. In [27], a protocol for the WPSN is proposed, and the characteristics of a full-duplex wireless-powered relay have been studied. The results have shown a fact that the throughput of the proposed protocol can be improved significantly when it is compared to the existing ones. In [29], authors have applied zero-forcing beamforming to optimize the energy harvesting capability and enhance the system performance. Given delay constraints, optimal stochastic power control for energy harvesting system has been investigated in [30-32]. Most recently, transmit power minimization for wireless networks with energy harvesting relays have been analyzed in [33].

Motivated by all above works, in this paper, we investigate two WPT MAC protocols, namely HHT and HDT, to improve the reliable communication of wireless sensor networks. Therein, the HHT protocol is derived from previous publications [17, 20, 22]. More specifically, we consider that multiple sensor nodes, which are widely used to measure temperature, pressure, humidity, etc., in industrial wireless networks, are scheduled to harvest the energy from a HAP following one of the considered protocols. Thereafter, they send their packets to the HAP following the assigned timeslot. The packet transmitted from the single node to the HAP should meet the strict deadline to satisfy the reliable communication requirements. To reduce the packet loss, the HAP can use either the MRC or the SC technique to process the received packet. Given these settings, the performance analysis of the considered system is investigated. The contributions and main results of this paper are summarized as follows:

- Two protocols, namely as HHT and HDT, for multiple sensor nodes of the wireless network by employing time division multiple access (TDMA) are investigated.

- We characterize two performance metrics for the considered system model which include: 1) packet timeout probability for uplink information transmission (ULIT) of a single node. 2) System reliability in terms of successful probability of packet transmission and system outage probability for the ULIT. These performance metrics are useful tools to provide a fast evaluation and parameter optimization of sensor installations.

- The numerical results are provided to compare the performance between the HHT and HDT protocols. By simply rearranging energy harvesting timeslots, the performance of the HDT protocol outperforms the one of the HHT protocol for both the MRC and SC techniques. Further, the obtained results can be 
extended to analyze the performance of multi-hop communication in the WPSN.

To the best of authors' knowledge, there is no previous publication studying this problem.

The remainders of this paper are presented as follows. In Sect. 2, the system model, assumptions, and WPT protocols for the sensor network are introduced. In Sect. 3, the performance metrics for a single sensor node and whole system are introduced. Accordingly, closed-form expressions for the packet outage probability, system reliability, and system outage probability are derived in Sect. 4. In Sect. 5, the numerical results and discussions are provided. Finally, the conclusion is given in Sect. 6.

\section{System model}

In this section, we introduce the system model, channel assumptions, and WPT schemes for the considered WPSN.

\subsection{System model}

Let us consider a WPSN as shown in Fig. 1 in which $K$ sensor nodes are scheduled to harvest the energy and send packets to the HAP. The HAP is assumed to have $M+1$ antennas in which one special antenna is designed for the downlink wireless energy transfer (DWET) and the $M$ antennas are used to receive the ULIT. Here, the HAP can employ either the SC or the MRC technique to process the received information. Due to the limited energy, the sources need to be charged by the HAP following a specific protocol (see Sects. 2.2 and 2.3). The channel gain of the

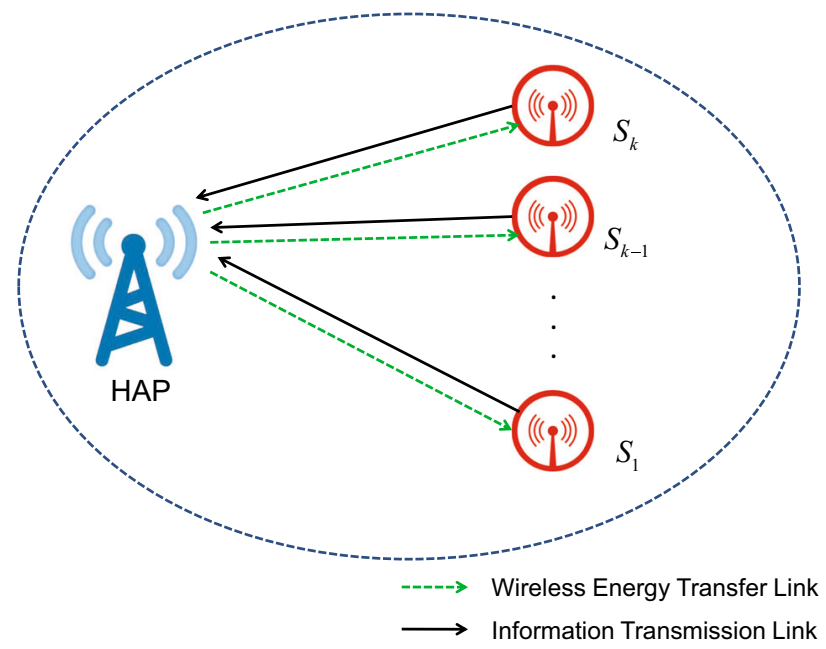

Fig. 1 A system model of wireless powered communications. There are $K$ nodes scheduled to harvest the energy and then communicate with the HAP. The green dash lines are DWET, while the black solid lines are ULIT (Color figure online)
ULIT from the sensor node $S_{k}$ to the $j$ th antenna of the HAP is denoted by $g_{k j}, j \in\{1,2, \ldots, M\}$. The channel gain of the DWET from the HAP to the sensor node $S_{k}$ is expressed by $f_{k}$ or $h_{k}, k \in\{1,2, \ldots, K\}$ depending on the energy harvesting protocol. Note that the sensors are often equipped with a battery to start the energy harvesting process and they also use such a battery as a backup energy source [10]. However, the analysis of the battery consumption is out of scope of this paper.

To make the line with recent publications [34-37], we assume that all channel coefficients are modeled as Rayleigh blocked flat fading, i.e., the channels remain constant during transmission of one packet but they may independently change thereafter. Accordingly, the channel gains are random variables (RVs) distributed following an exponential distribution, and the probability density function (PDF) and cumulative distribution function (CDF) are formulated, respectively, as,

$f_{X}(x)=\frac{1}{\Omega_{X}} \exp \left(-\frac{x}{\Omega_{X}}\right)$,

$F_{X}(x)=1-\exp \left(-\frac{x}{\Omega_{X}}\right)$,

where $X$ is the $\mathrm{RV}$ refers to the channel gain, and $\Omega_{X}=$ $\mathbf{E}[X]$ is the channel mean gain. In the considered system, the channel mean gains of RVs $f_{k}, h_{k}$, and $g_{k j}$ are denoted by $\Psi_{k}, \Omega_{k}$, and $\beta_{k}$, respectively.

\subsection{HHT protocol}

This protocol employs the TDMA approach as shown in Fig. 2 in which a time block $T$ is separated into $K$ timeslots, and each timeslot is assigned for one sensor node $S_{k}$ with the period of $\left(t_{0 k}+t_{k}\right) T$. Here, the header of each timeslot $t_{0 k} T$ is used for the DWET while the remainder $t_{k} T$ is dedicated to send the data packet to the HAP. In other words, the total time block of the energy harvesting and information transfer can be expressed as

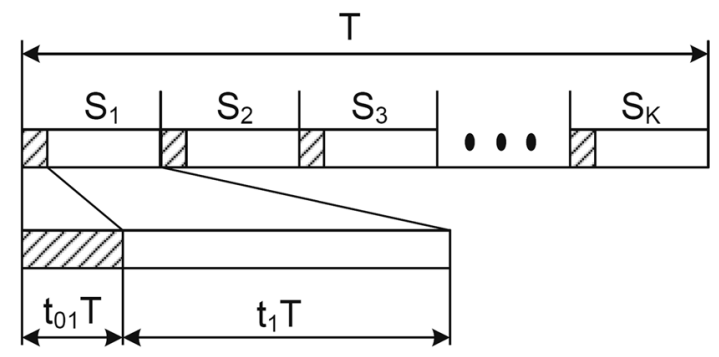

Fig. 2 The timeslot of each sensor node $S_{k}$ is devised by two subtimeslots, $t_{0 k} T$ and $t_{k} T$. The sub-timeslot $t_{0 k} T$ is used for the DWET while the sub-timeslot $t_{k}$ is used for the ULIT 
$\sum_{k=1}^{K} t_{0 k} T+\sum_{k=1}^{K} t_{k} T=T$

i.e., $\sum_{\tau=0}^{K} t_{\tau}=1, t_{\tau}$ is a fraction of timeslot $T$ which satisfies $0<t_{\tau}<1$, and $t_{0}$ denotes the total time for the energy harvesting, defined by

$t_{0}=\sum_{k=1}^{K} t_{0 k}, \quad 0<t_{0 k}<1, \quad \forall k \in\{1,2, \ldots, K\}$,

In the period $t_{0 k} T$, the HAP uses one special antenna to transfer the energy to the sensor node $S_{k}$, and hence the harvested energy at the $S_{k}$ can be expressed as follows

$E_{k}=\eta_{k} t_{0 k} T P f_{k} d_{k}^{-\alpha}, \quad \forall k \in\{1,2, \ldots, K\}$,

where $P$ is the transmission power of the HAP, $d_{k}$ is distance from the HAP to the node $S_{k}, \alpha$ is the path-loss exponent, and $\eta_{k} \in(0,1)$ is the energy conversion efficiency coefficient which depends on the harvesting circuitry [38, 39]. After energy harvesting period, the sensor node $S_{k}$ uses its harvested energy to send the data packet to the HAP with the power given by

$P_{k}=\frac{E_{k}}{t_{k} T}=\frac{\eta_{k} t_{0 k} P f_{k} d_{k}^{-\alpha}}{t_{k}}, \quad \forall k \in\{1 \ldots K\}$.

Moreover, the transmission time of the sensor node $S_{k}$ for one packet with size of $L$ bits can be formulated as the ratio of packet size to the transmission rate as follows [40-44]

$T_{k}^{(v)}=\frac{\widetilde{B}_{k}}{\ln \left(1+\rho_{k} \gamma_{k}^{(v)}\right)}, \quad \forall k \in\{1,2, \ldots, K\}$,

where $\widetilde{B}_{k}=\frac{L \ln (2)}{W t_{k}}, W$ is the system bandwidth, and $\rho_{k}$ is a constant related to a specific target bit error rate of M-ary quadrature amplitude modulation (MQAM), $\rho_{k}=$ $-\frac{1}{\log \left(5 * B E R_{k}\right)}[45,46]$. Accordingly, the SNR $\gamma_{k}^{(v)}$ can be expressed as (see Appendix 1)

$\gamma_{k}^{(v)}=\frac{P_{k} g_{k}^{(v)} d_{k}^{-\alpha}}{W N_{0}}=\frac{\eta_{k} t_{0 k} \gamma_{0} f_{k} g_{k}^{(v)} d_{k}^{-2 \alpha}}{t_{k}}$,

where $\gamma_{0}=\frac{P}{W N_{0}}, v \in\{S C, M R C\}$ technique, and if the HAP employs the SC technique, i.e. $v=S C, g_{k}^{(v)}$ can be formulated as

$g_{k}^{(S C)}=\max _{j \in\{1,2, \ldots, M\}}\left\{g_{k j}\right\}$,

otherwise if the HAP uses the MRC technique to process the received signal, i.e. $v=M R C$, then $g_{k}^{(v)}$ can be given by

$g_{k}^{(M R C)}=\sum_{j=1}^{M} g_{k j}$

\subsection{HDT protocol}

In contrast with the HHT protocol, in the HDT protocol, the HAP uses a dedicated timeslot, $t_{0}$, at the beginning of the block to harvest the energy, while other timeslots are only used for ULIT (see Fig. 3). Accordingly, the sensor node $S_{k}$ can harvest the energy over the downlink $h_{k}$ as

$E_{k}=\eta_{k} P d_{k}^{-\alpha} h_{k} t_{0} T, \quad \forall k \in\{1,2, \ldots, K\}$.

After the energy harvesting period, the sensor nodes wait for their assigned timeslot to send the packet to the HAP with the power given by

$P_{k}=\frac{E_{k}}{t_{k} T}=\frac{t_{0} \eta_{k} h_{k} P d_{k}^{-2 \alpha}}{t_{k}}, \quad \forall k \in\{1 \ldots K\}$.

Accordingly, the SNR at the HAP when the sensor node $S_{k}$ used its harvested energy to transmit the packet to the HAP is given as

$\gamma_{k}^{(v)}=\frac{P_{k} g_{k} d_{k}^{-\alpha}}{W N_{0}}=\frac{\eta_{k} t_{0} \gamma_{0} h_{k} g_{k}^{(v)} d_{k}^{-2 \alpha}}{t_{k}}$.

In this protocol, the total time including the wireless energy transfer and communication transmission time for one block is also normalized to one as given in (3).

\section{Performance metrics}

In this section, we introduce performance metrics for a single user and for a complete system.

\subsection{Packet timeout probability for the point-to-point communication}

When one packet from the sensor node $S_{k}$ is sent to the HAP in its assigned timeslot, it may be timed out or erroneous due to channel impairment. The event of successful transmission is given as $T_{k, \text { succ }}^{(v)}=\left\{T_{k}^{(v)} \mid T_{k}^{(v)}<t_{\text {out }, k}\right\}$ where $T_{k}^{(v)}$ is defined in (7), and $t_{\text {out }, k}$ is the threshold for the transmission time of one packet. Clearly, the packet transmission time is a function of random variables which depend on the channel state information (CSI) of the energy harvesting phase and the information transmission phase. According to the probability definition, the CDF of

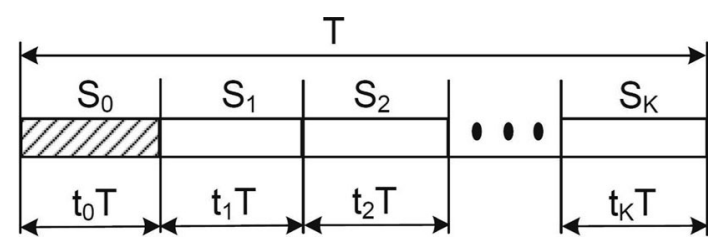

Fig. 3 The dedicated timeslot $t_{0} T$ is used to transfer the energy to all sources, the other timeslots $t_{k} T$ are assigned to the source $S_{k}$ 
the packet transmission time for the sensor node $S_{k}$ can be formulated as

$$
F_{T_{k}^{(v)}}(t)=\operatorname{Pr}\left\{T_{k}^{(v)}<t\right\} .
$$

Further, the packet timeout probability is defined as the probability that the packet transmission time is greater than the timeout threshold, $t_{\text {out }, k}$. In other words, this probability can be expressed as

$$
\mathcal{O}_{k}^{(v)}=\operatorname{Pr}\left\{T_{k}^{(v)} \geq t_{\text {out }, k}\right\}=1-F_{T_{k}^{(v)}}\left(t_{\text {out }, k}\right) .
$$

Without loss of generality, we set $t_{\text {out }, k}=t_{k} T=t_{\text {out }}$, i.e., the threshold for the transmission time of one packet is equal to the period of one time slot, and consider the performance metrics as follows.

\subsection{System performance metrics}

To evaluate the system performance, we introduce two metrics, namely system reliability and outage probability.

\subsubsection{System reliability}

The system reliability of the WPSN is defined as the probability of the packet transmission time for the node having the worst channel condition and still satisfying the timeout threshold, given as

$\overline{\mathbf{R}}^{(v)}=\operatorname{Pr}\left\{T_{\max }^{(v)}<t_{\text {out }}\right\}$,

where $T_{\max }^{(v)}=\max _{k \in\{1,2, \ldots, K\}}\left\{T_{k}^{(v)}\right\}$.

\subsubsection{Outage probability}

Outage probability is defined as the probability of the packet transmission time for the node having the best channel condition but not satisfying the timeout threshold, given as

$\mathcal{O}_{\text {sys }}^{(v)}=\operatorname{Pr}\left\{T_{\min }^{(v)} \geq t_{\text {out }}\right\}$.

where $T_{\min }^{(v)}=\min _{k \in\{}\left\{T_{k}^{(v)}\right\}$. To investigate further, let us consider an importänin lemma as follows.

Lemma 1 Assume that the $R V X_{k}$ and $Y_{k j}$ are independent and distributed following an exponential distribution with mean values $\Omega_{k}$ and $\beta_{k}$, respectively. We define a new $R V$ as $Z_{k}^{(v)}=X_{k} Y_{k}^{(v)}$ where

$$
Y_{k}^{(v)}=\left\{\begin{array}{cc}
\sum_{j=1}^{M} Y_{k j}, & v=M R C \\
\max _{j \in\{1,2, \ldots, M\}}\left\{Y_{k j}\right\}, & v=S C
\end{array} .\right.
$$

The CDF of the $R V Z_{k}^{(v)}, v \in\{M R C, S C\}$ is formulated as follows

$$
F_{Z_{k}^{(v)}}(z)=\left\{\begin{array}{cc}
(20), & v=M R C \\
(21), & v=S C
\end{array},\right.
$$

in which

$$
\begin{aligned}
F_{Z_{k}^{(M R C)}}(z) & =1-\frac{2}{\Gamma(M)}\left(\frac{z}{\Omega_{k} \beta_{k}}\right)^{\frac{M}{2}} K_{M}\left(2 \sqrt{\frac{z}{\Omega_{k} \beta_{k}}}\right) \\
F_{Z_{k}^{(S C)}}(z) & =1-2 M \sum_{m=0}^{M-1}\left(\begin{array}{c}
M-1 \\
m
\end{array}\right)(-1)^{m} \\
& \times \sqrt{\frac{z}{(m+1) \beta_{k} \Omega_{k}}} K_{1}\left(2 \sqrt{\frac{(m+1) z}{\Omega_{k} \beta_{k}}}\right) .
\end{aligned}
$$

where $\Gamma(\cdot)$ and $K_{m}(x)$ denote Gamma function [47, Eq. (8.339.1)] and modified Bessel function [47, Eq. (3.471.9)], respectively.

Proof The proof is provided in "Appendix 1".

\section{Performance analysis}

In the following section, we use the results of Lemma 1 to analyze the performance of the considered system for both HDT and HHT protocols.

\subsection{Analysis of the HHT protocol}

\subsubsection{Packet timeout probability for a single node}

To derive the packet timeout probability for node $S_{k}$, we first derive the CDF of packet transmission time by combining (7) with (8), and the expression (14) can be rewritten as follows

$$
F_{T_{k}^{(v)}}(t)=1-\operatorname{Pr}\left\{f_{k} g_{k}^{(v)}<\left[\exp \left(\frac{\widetilde{B}_{k}}{t}\right)-1\right] \mathcal{A}_{k}\right\},
$$

where $\mathcal{A}_{k}=\frac{t_{k} d_{k}^{2 \alpha}}{\gamma_{0} \eta_{k} \rho_{k} t_{0 k}}$. Using Lemma 1 , the CDF of $T_{k}^{(v)}$ in the MRC and SC schemes can be obtain easily as follows:

$$
\begin{aligned}
F_{T_{k}^{(M R C)}}(t) & =\frac{2}{\Gamma(M)}\left(\frac{\Upsilon(t) \mathcal{A}_{k}}{\Psi_{k} \beta_{k}}\right)^{\frac{M}{2}} K_{M}\left(2 \sqrt{\frac{\Upsilon(t) \mathcal{A}_{k}}{\Psi_{k} \beta_{k}}}\right), \\
F_{T_{k}^{(S C)}}(t) & =2 M \sum_{m=0}^{M-1}\left(\begin{array}{c}
M-1 \\
m
\end{array}\right)(-1)^{m} \\
& \times \sqrt{\frac{\Upsilon(t) \mathcal{A}_{k}}{(m+1) \beta_{k} \Psi_{k}}} K_{1}\left(2 \sqrt{\frac{(m+1) \Upsilon(t) \mathcal{A}_{k}}{\Psi_{k} \beta_{k}}}\right),
\end{aligned}
$$

where $\Upsilon(t)=\exp \left(\frac{\widetilde{B_{k}}}{t}\right)-1$ 
From (15), we derive the packet outage probability of the MRC and SC schemes by using (23) and (24) as follows:

$$
\begin{aligned}
& \mathcal{O}_{k}^{(M R C)}=1-F_{T_{k}^{(M R C)}}\left(t_{\text {out }}\right), \\
& \mathcal{O}_{k}^{(S C)}=1-F_{T_{k}^{(M R C)}}\left(t_{\text {out }}\right) .
\end{aligned}
$$

\subsubsection{System performance of the HHT scheme}

Since the packet transmission time of each node is independent, the CDF of $T_{\max }^{(v)}$ and $T_{\min }^{(v)}$ can be obtained by using the order statistics theory as follows:

$$
\begin{aligned}
F_{T_{\max }^{(v)}}(t) & =\operatorname{Pr}\left\{\max _{k \in\{1,2, \ldots, K\}}\left\{T_{k}^{(v)}\right\}<t\right\} \\
& =\prod_{k=1}^{K} F_{T_{k}^{(v)}}(t), \\
F_{T_{\min }^{(v)}}(t) & =\operatorname{Pr}\left\{\min _{k \in\{1,2, \ldots, K\}}\left\{T_{k}^{(v)}\right\}<t\right\} \\
& =1-\prod_{k=1}^{K}\left(1-F_{T_{k}^{(v)}}(t)\right) .
\end{aligned}
$$

System reliability Following the definition given in (16) and using (27), we obtain the system reliability for the HHT scheme as follows

$\overline{\mathbf{R}}^{(v)}=F_{T_{\max }^{(v)}}\left(t_{\text {out }}\right)=\prod_{k=1}^{K} F_{T_{k}^{(v)}}\left(t_{\text {out }}\right)$,

where $v \in\{M R C, S C\}, F_{T_{k}^{(M R C)}}(t)$ and $F_{T_{k}^{(S C)}}(t)$ are defined in (23) and (24), respectively.

Outage probability According to the definition of the outage probability given in (17) and using (28), the closedform expression for the outage probability can be easily derived as

$$
\begin{aligned}
\mathcal{O}_{\text {sys }}^{(v)} & =1-F_{T_{\min }^{(v)}}\left(t_{\text {out }}\right) \\
& =\prod_{k=1}^{K}\left(1-F_{T_{k}^{(v)}}\left(t_{\text {out }}\right)\right), \quad v \in\{M R C, S C\},
\end{aligned}
$$

where $F_{T_{k}^{(M R C)}}(t)$ and $F_{T_{k}^{(S C)}}(t)$ are formulated in (23) and (24), respectively.

\subsection{Analysis of the HDT protocol}

\subsubsection{Packet timeout probability for a single node}

Similar to the HHT protocol, we need to derive the CDF of packet transmission time for the sensor node operating in the HDT protocol. In particular, the CDF of $T_{k}^{(v)}$ in the
HDT scheme can be rewritten by combining (13) with (7) and using (14) as

$F_{T_{k}^{(v)}}(t)=\operatorname{Pr}\left\{h_{k} g_{k}^{(v)} \leq \Upsilon(t) \mathcal{B}_{k}\right\}$

where $\mathcal{B}_{k}=\frac{t_{k} d_{k}^{2 \alpha}}{\gamma_{0} \eta_{k} \rho_{k} t_{0}}$. It is easy to see that the final expression for $F_{T_{k}^{(v)}}(t)$ in (31) can be obtained by using Lemma 1. In particular, the CDF of $F_{T_{k}^{(v)}}(t)$ in the HDT scheme are given, respectively, as

$$
\begin{aligned}
F_{T_{k}^{(M R C)}}(t) & =\frac{2}{\Gamma(M)}\left(\frac{\Upsilon(t) \mathcal{B}_{k}}{\Omega_{k} \beta_{k}}\right)^{\frac{M}{2}} K_{M}\left(2 \sqrt{\frac{\Upsilon(t) \mathcal{B}_{k}}{\Omega_{k} \beta_{k}}}\right), \\
F_{T_{k}^{(S C)}}(t) & =2 M \sum_{m=0}^{M-1}\left(\begin{array}{c}
M-1 \\
m
\end{array}\right)(-1)^{m} \\
& \times \sqrt{\frac{\Upsilon(t) \mathcal{B}_{k}}{(m+1) \beta_{k} \Omega_{k}}} K_{1}\left(2 \sqrt{\frac{(m+1) \Upsilon(t) \mathcal{B}_{k}}{\Omega_{k} \beta_{k}}}\right) .
\end{aligned}
$$

Accordingly, the packet outage probability of a single node can be obtained by using (32) and (33) as follows:

$\mathcal{O}_{k}^{(M R C)}=1-F_{T_{k}^{(M R C)}}\left(t_{\text {out }}\right)$,

$\mathcal{O}_{k}^{(S C)}=1-F_{T_{k}^{(S C)}}\left(t_{\text {out }}\right)$.

where $F_{T_{k}^{(M R C)}}(t)$ and $F_{T_{k}^{(S C)}}(t)$ are defined in (32) and (33), respectively.

\subsubsection{System performance of the HDT protocol}

To make a comparison of the system performance between the HHT and HDT protocols, we derive the system reliability and outage probability.

System reliability According to (16), we can express the system reliability for the HDT protocol as

$\overline{\mathbf{R}}^{(v)}=F_{T_{\max }^{(v)}}\left(t_{\text {out }}\right)=\prod_{k=1}^{K} F_{T_{k}^{(v)}}\left(t_{\text {out }}\right)$,

where $F_{T_{k}^{(M R C)}}(t)$ and $F_{T_{k}^{(S C)}}(t)$ are formulated in (32) and (33), respectively.

Outage probability From (17), we can derive the outage probability for the considered WPSN by using order statistics theory as follows

$$
\begin{aligned}
\mathcal{O}_{\text {sys }}^{(v)} & =1-F_{T_{\min }^{(v)}}\left(t_{\text {out }}\right) \\
& =\prod_{k=1}^{K}\left(1-F_{T_{k}^{(v)}}\left(t_{\text {out }}\right)\right), \quad v \in\{M R C, S C\},
\end{aligned}
$$

where $F_{T_{k}^{(M R C)}}(t)$ and $F_{T_{k}^{(S C)}}(t)$ are defined in (32) and (33), respectively. 


\section{Numerical results}

In this section, analytical and simulation results for the considered WPSN are presented. More specifically, we compare the performance of a single node and the system with respect to the HDT and HHT protocols. We consider a 2-D simulation setup, where $\left(x_{k}, y_{k}\right)$ is the coordinate of $k-$ sensor node, and $k=0$ is for the HAP. We use the MonteCarlo simulation method with $10^{6}$ loops. Simulation algorithms for HHT and HDT protocols are presented in Algorithm 2 and 3, respectively. Unless otherwise stated, the following system parameters are set as follows:

- Packet size: $L=128$ bytes [48];

- Timeout threshold: $t_{\text {out }}=0.864$ miliseconds [48];

- Bit error rate: $\mathrm{BER}_{k}=10^{-2}$;

- Channel mean gains: $\Psi_{k}=\Omega_{k}=\beta_{k}=1$;

- Transmit SNR of the HAP: $\gamma_{0}=\frac{P}{W N_{0}}$;

- The HAP locates at: $\left(x_{0}, y_{0}\right)=(0,0)$;

- The pathloss exponent $\alpha=4$;

- System bandwidth: $W=2 \mathrm{MHz}$;

Figure 4 analyzes the impact of non-identical distances on the packet timeout probability of individual sensor nodes. We can observe from Fig. 4 that node 4 has the lowest packet timeout probability, i.e., it obtains the best performance over all nodes. This is because the distance between the HAP and the sensor node 4 is the shortest, i.e., $d_{4}=0.14$, hence it can harvest the energy, and transfers the information to the HAP better than other nodes. At a specific node, e.g. node 1 , we can see that the packet timeout probability of the HDT protocol is always smaller than the one of HHT protocol. The sensor node using HDT protocol together with MRC technique obtains the best performance. Furthermore, we can observe from Fig. 5 that given the same system parameters, i.e. $d_{k}=0.7$, which

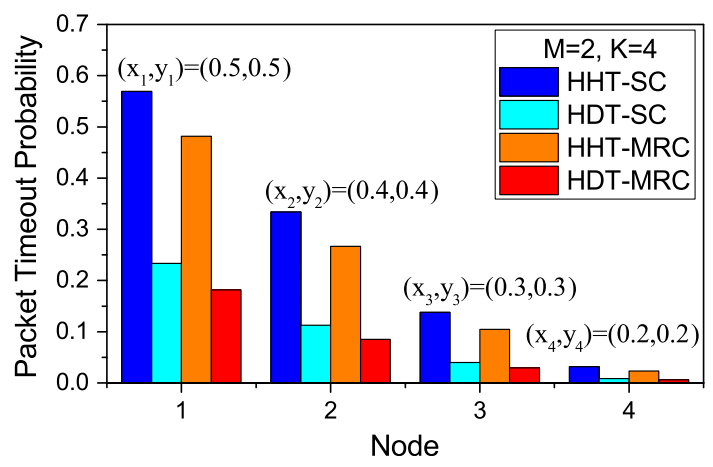

Fig. 4 Analytical results of packet timeout probability versus nonidentical distances between the sensor node $S_{k}$ and the HAP. The harvesting time in the HDT and HHT are $t_{0 k}=0.05$ and $t_{0}=\sum_{k=1}^{K=4} t_{0 k}=0.2$, respectively. Information transmission time for all nodes is set to $t_{k}=0.2$, the energy harvesting efficiency coefficient $\eta_{k}=0.5$, and the SNR is set to $\gamma_{0}=5 \mathrm{~dB}$ sensor node has a higher value of the energy harvesting efficiency coefficient, e.g., $\eta_{4}=0.9$, it will provide a better performance. Also, the packet timeout probability of the HDT protocol is always smaller than the one of the HHT protocol at the specific sensor node. It means that the performance of the HDT protocol is always better than the one of the HHT protocol.

Figure 6 shows a comparison of the system reliability between the HHT and HDT protocols. Sensor nodes are located at the same position as $\left(x_{k}, y_{k}\right)=(0.3,0.3)$. As can clearly be seen from Fig. 6, the simulation and analysis results match well for all protocols. More specifically, the system reliability is increased as the transmit SNR $\gamma_{0}$ of the HAP increases. This is because that the HAP can transfer a strong energy to the farthest sensor node as the transmit SNR $\gamma_{0}$ increases, i.e., the sensor nodes can harvest more energy. Accordingly, they can transmit the information back to the HAP with a higher power level. Therefore, the

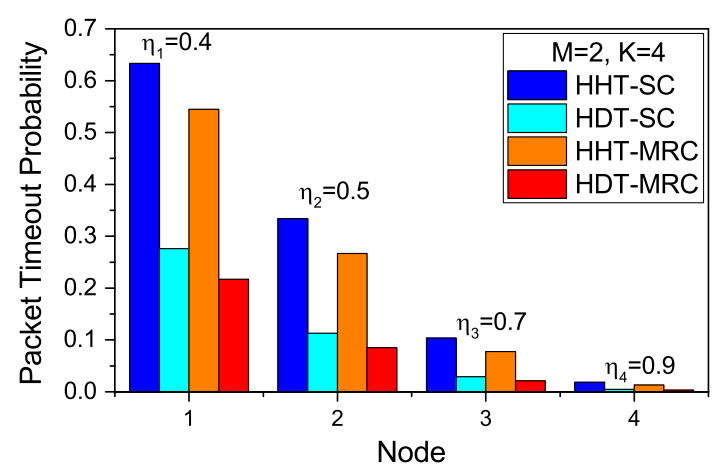

Fig. 5 Analytical results of packet timeout probability versus nonidentical energy harvesting efficiency coefficients. The HAP and $S_{k}$ have $M=2$ and $K=4$ antennas, respectively. The harvesting time in the HDT and HHT are $t_{0 k}=0.05$ and $t_{0}=\sum_{k=1}^{K=4} t_{0 k}=0.2$, respectively. Information transmission time for all nodes is set to $t_{k}=0.2$, and the SNR is set to $\gamma_{0}=5 \mathrm{~dB}$

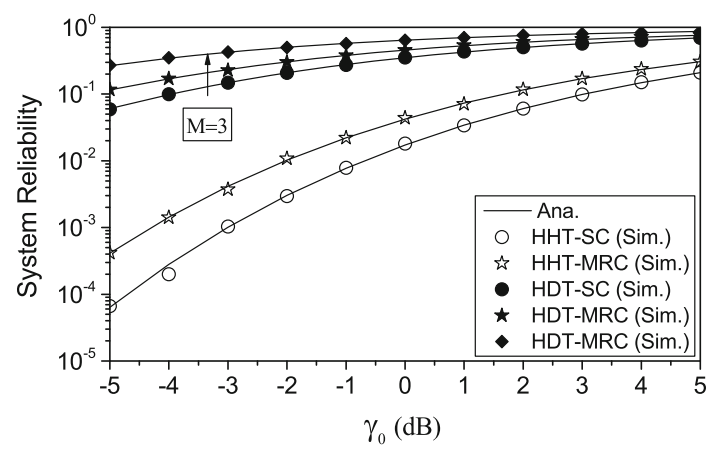

Fig. 6 System reliability versus the transmission power of the HAP in which the HAP has $M=2$ antennas. The harvesting time in the HDT and HHT are $t_{0 k}=0.01$ and $t_{0}=\sum_{k=1}^{K=5} t_{0 k}=0.05$, respectively. Information transmission time for all nodes is set to $t_{k}=0.19$, the energy harvesting efficiency coefficient $\eta_{k}=0.5$ 
received SNR at the HAP is improved, i.e., the reliability is improved. Further, the system reliability of the HDT protocol outperforms the one of the HHT in both SC and MRC techniques. It is due to the fact that the total time used for the DWET in the HDT protocol is greater than the one of the HHT protocol. Accordingly, the nodes in the HDT protocol can harvest more energy from the HAP, and hence they can transmit with a higher power level, i.e, the reliability is enhanced. Further, in the transmission SNR regime $\gamma_{0} \geq-1 \mathrm{~dB}$, the system reliability is rather high (greater than 90\%). This means that the HDT scheme with the MRC technique may be a potential solution for wireless applications where stringent delay and reliable communication are the most important criteria. We also can observe that when the number of antennas at the HAP increases from $M=2$ to $M=3$, the system reliability is improved significantly. It is easy to understand that increasing the number of antennas leads to an enhanced received signal at the HAP, i.e., the probability of successful receiving packet is improved.

In Fig. 7, the impact of length of energy harvesting timeslot on the performance of HHT and HDT protocols is presented, the HAP is assumed to use the MRC technique to process the received signal. Firstly, we observe the performance of HHT protocol as $t_{0 k}$ increases from 0.01 to 0.05 ( $t_{0}$ is increased from 0.05 to 0.25 ). It is clear to see that the system reliability is improved significantly, as the length of energy harvesting time increases. This is because that increasing energy harvesting time leads to increase the energy harvested at sensors, i.e., increases the transmission power for all sensors. Accordingly, the probability of a packet being timeout is reduced, i.e., the system reliability is increased. However, as $t_{0 k}$ increases further, i.e., $t_{0 k}=$ $0.1\left(t_{0}=0.5\right)$, the system reliability is degraded when it is compared to $t_{0 k}=0.01,0.05$. This is due to the fact that as

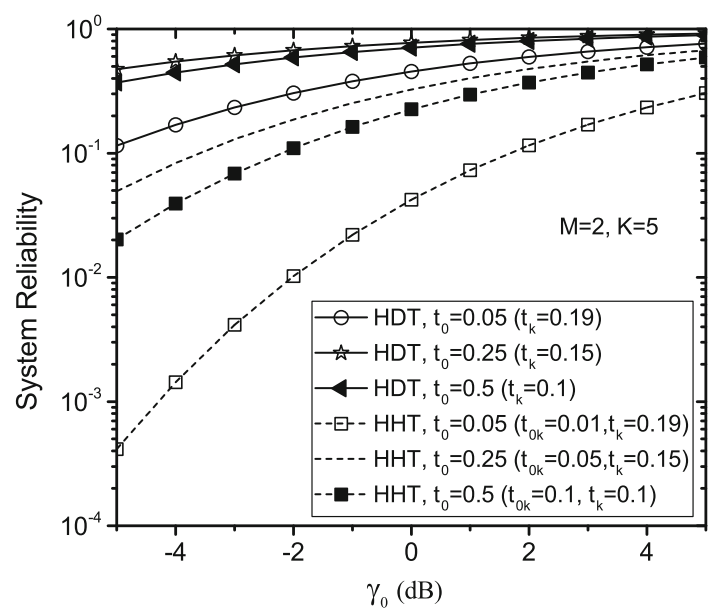

Fig. 7 Impact of energy harvesting timeslot length on the system reliability. The HAP using MRC technique has $M=2$ antennas. The energy harvesting efficiency coefficient is set to $\eta_{k}=0.5$ the major time is used to harvest the energy, the remaining timeslot used to transmit packet is very short. Consequently, the probability that a packet is dropped due to a timeout increases, i.e., the system reliability is degraded. Secondly, we observe the same behaviors for HDT protocol when the energy harvesting time $t_{0}$ is increased. It is easy to see that the system reliability of the HDT is improved as $t_{0}$ increases from from 0.05 to 0.25 , and decreases as $t_{0}=0.5$. This is due to the same reason as in the HHT protocol, i.e., if the length of energy harvesting timeslot is reasonable, the system performance is improved significantly, otherwise it is degraded. Finally, we see that the HDT protocol outperforms the one of the HHT protocol under the same energy harvesting time $t_{0}$.

In Fig. 8, we examine the impact of the energy harvesting efficiency coefficient, $\eta_{k}$, on the outage probability of the considered WPSN, where sensor nodes are located at the same position $\left(x_{k}, y_{k}\right)=(0.65,0.65), k=1, \ldots, 5$. It is clear to see that the simulation and analytical results match very well. Specifically, the outage probability decreases when the energy harvesting efficiency coefficient, $\eta_{k}$, increases as expected. This can be understood that sensor nodes can harvest more energy when the energy harvesting efficiency coefficient increases, the energy harvesting capability of circuit in the sensor nodes is improved. Accordingly, the sensor nodes can transmit packets to the HAP with a high power level, i.e., the packet timeout probability is decreased. Furthermore, we can see that the outage probability curves of the HDT protocol are always below the ones of the HHT protocol for both the SC and MRC techniques. These results fit with the discussions in Fig. 6, i.e., the performance of HDT scheme outperforms the one of the HHT protocol. Moreover, the outage probability in the HDT with the MRC technique decreases significantly as the number of antennas at the HAP

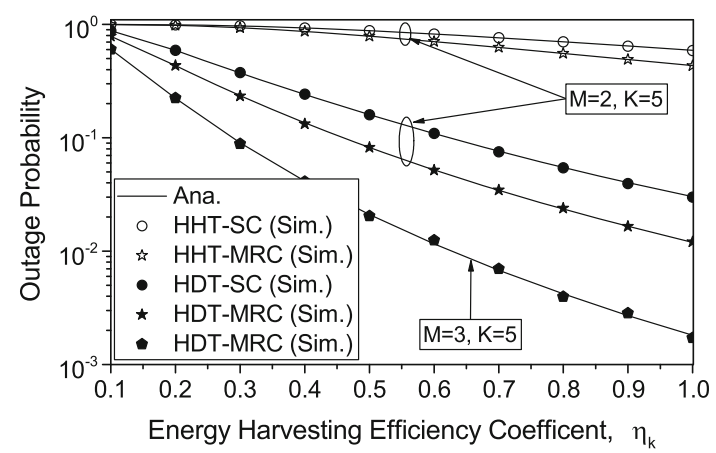

Fig. 8 Outage probability versus the energy harvesting efficiency coefficient $\eta_{k}$. The energy harvesting time in the HDT and HHT are $t_{0 k}=0.01$ and $t_{0}=\sum_{k=1}^{K=5} t_{0 k}=0.05$, respectively. Information transmission time for all nodes is set to $t_{k}=0.19$, the HAP transmission power is set to $\gamma_{0}=5(\mathrm{~dB})$, and $\left(x_{k}, y_{k}\right)=(0.45,0.45)$ 


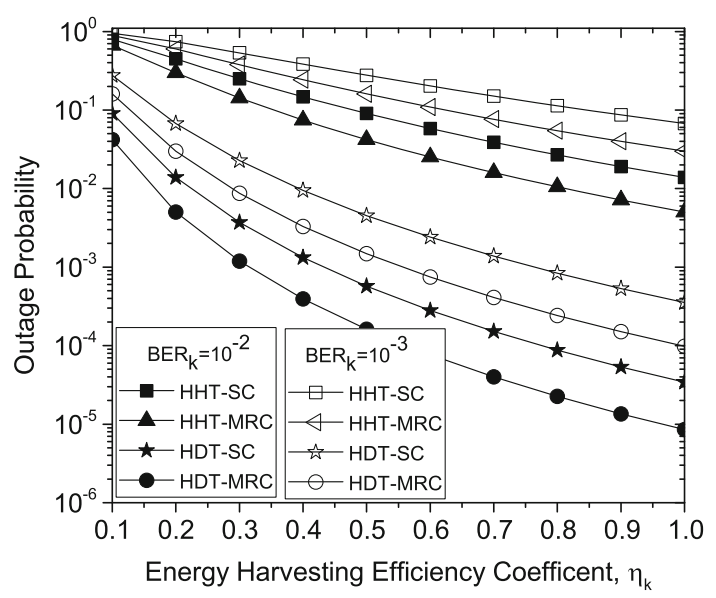

Fig. 9 Analytical results of outage probability versus the energy harvesting efficiency coefficient $\eta_{k}$ with $\mathrm{BER}_{k}=\left\{10^{-2}, 10^{-3}\right\}$. The energy harvesting time in the HDT and HHT are $t_{0 k}=0.01$ and $t_{0}=\sum_{k=1}^{K=5} t_{0 k}=0.05$, respectively. Information transmission time for all nodes is set to $t_{k}=0.19$, and the HAP transmission power $\gamma_{0}=5(\mathrm{~dB})$

increases from $M=2$ to the $M=3$. This can be explained by the fact that the increasing the number of antennas at the HAP leads to enhance the signal strength at the HAP, the packet transmission time and erroneous packets are reduced. As a result, the outage probability decreases, or the system performance is improved. As increasing the demand on bit error rate target from $\mathrm{BER}_{k}=10^{-2}$ to $\mathrm{BER}_{k}=10^{-3}$ as in Fig. 9, the HDT protocol with MRC technique still obtains the best performance among all combinations, In other words, the HDT with MRC technique is a reason approach to guarantee the reliability in the wireless energy harvesting networks.

\section{Conclusion}

In this paper, two WPT protocols for wireless sensor networks were proposed and compared. More specifically, the analytical expressions of the packet time probability and reliable communication for the proposed protocols over Rayleigh fading channels are derived. These obtained performance metrics were subsequently used to compare the performance of proposed protocols with respect to the $\mathrm{SC}$ and MRC techniques. Further, these obtained expressions can be useful tools for a fast evaluation and parameter optimization of the WPSN implementations. Numerical examples showed that the proposed HDT protocol using the MRC technique outperforms all other simulated scenarios. The performance of the proposed HDT protocol can be further improved when the number of antennas at the HAP increases. Thus, the HDT protocol using MRC technique can be a promising solution for wireless sensor networks with high reliability demands. In the future research, we will utilize the advantage of RF energy harvesting and study the performance of multi-hop communication and the impact of interference on communication links with high reliability demands.

Acknowledgements The research leading to these results has been performed in the SafeCOP project which is funded from the ECSEL Joint Undertaking under grant agreement n0 692529, and from National funding.

Open Access This article is distributed under the terms of the Creative Commons Attribution 4.0 International License (http://crea tivecommons.org/licenses/by/4.0/), which permits unrestricted use, distribution, and reproduction in any medium, provided you give appropriate credit to the original author(s) and the source, provide a link to the Creative Commons license, and indicate if changes were made.

\section{Appendix}

\section{Proof for Lemma 1}

To prove Lemma 1, we should find the CDF of $Z_{k}^{(M R C)}$ and $Z_{k}^{(S C)}$ as following.

\section{The CDF of $Z_{k}^{(M R C)}$}

According to the conditional probability, the CDF of $Z_{k}^{(M R C)}$ can be formulated as

$$
\begin{aligned}
F_{Z_{k}^{(M R C)}}(z) & =\operatorname{Pr}\left\{X_{k} Y_{k}^{(M R C)}<z\right\} \\
& =\int_{0}^{\infty} \operatorname{Pr}\left\{X_{k}<\frac{z}{y}\right\} f_{Y_{k}^{(M R C)}}(y) d y .
\end{aligned}
$$

Further, we know that $Y_{k}^{M R C}=\sum_{j=1}^{M} Y_{k j}$ are the sum of $M$ independent $\mathrm{RVs}$ distributed following an exponential distribution. Thus, the CDF of $Y_{k}^{M R C}$ is an incomplete Gamma function as

$$
F_{Y_{k}^{(M R C)}}(y)=\operatorname{Pr}\left\{\sum_{j=1}^{M} Y_{k j}<y\right\}=1-\frac{\Gamma\left(M, \frac{y}{\beta_{k}}\right)}{\Gamma(M)} .
$$

Taking differentiation with respect to $y$ for (39) yields the PDF of $Y_{k}^{(M R C)}$ as

$f_{Y_{k}^{(M R C)}}(y)=\frac{y^{M-1}}{\beta_{k}^{M} \Gamma(M)} \exp \left(-\frac{y}{\beta_{k}}\right)$.

Also, we know that the CDF of $X_{k}$ is an exponential function as 
$F_{X_{k}}(x)=1-\exp \left(-\frac{x}{\Omega_{k}}\right)$

As a result, the expression in (38) can be rewritten by using (40) and (41) as follows

$F_{Z_{k}^{(M R C)}}(z)=1-\frac{1}{\beta_{k}^{M} \Gamma(M)} \int_{0}^{\infty} y^{M-1} \exp \left(\frac{z}{y \Omega_{k}}-\frac{y}{\beta_{k}}\right) d y$.

Using the help of [47, Eq. (3.471.9)] for the integral in (42), we finally obtain the CDF of $Z_{k}^{(M R C)}$ as in (20).

The CDF of $Z_{k}^{(S C)}$

In the $\mathrm{SC}$ scheme, the CDF of the $\mathrm{RV} Z_{k}^{(S C)}$ is expressed as $F_{Z_{k}^{(S C)}}(z)=\int_{0}^{\infty} \operatorname{Pr}\left\{X_{k}<\frac{z}{y}\right\} f_{Y_{k}^{(S C)}}(y) d y$,

where $Y_{k}^{(S C)}=\max _{j \in\{1,2, \ldots, M\}}\left\{Y_{k j}\right\}$ and the CDF of the RV $Y_{k j}$ is an exponential random variable, given by

$F_{Y_{k j}}(z)=1-\exp \left(-\frac{y}{\beta_{k}}\right)$.

Furthermore, the CDF and PDF of the $\mathrm{RV} Y_{k}^{(S C)}$ are derived, respectively, as follows:

$$
\begin{aligned}
F_{Y_{k}^{(S C)}}(y) & =\operatorname{Pr}\left\{\max _{j \in\{1,2, \ldots, M\}}\left\{Y_{k j}\right\}<y\right\}=\prod_{j=1}^{M} F_{Y_{k j}}(y) \\
& =\left[1-\exp \left(-\frac{y}{\beta_{k}}\right)\right]^{M}, \\
f_{Y_{k}^{(S C)}}(y) & =M f_{Y_{k j}}(y)\left[F_{Y_{k j}}(y)\right]^{M-1} \\
& =\sum_{m=0}^{M-1}\left(\begin{array}{c}
M-1 \\
m
\end{array}\right) \frac{M(-1)^{m}}{\beta_{k}} \exp \left(-\frac{(m+1) y}{\beta_{k}}\right) .
\end{aligned}
$$

Also, we have

$$
\operatorname{Pr}\left\{X_{k}<\frac{z}{y}\right\}=1-\exp \left(-\frac{z}{y \Omega_{k}}\right) .
$$

Substituting (46) and (47) into (48), we have

$$
\begin{aligned}
F_{Z_{k}^{(S C)}}(z) & =1-\sum_{m=0}^{M-1}\left(\begin{array}{c}
M-1 \\
m
\end{array}\right) \frac{M(-1)^{m}}{\beta_{k}} \\
& \times \int_{0}^{\infty} \exp \left(-\frac{z}{y \Omega_{k}}-\frac{(m+1) y}{\beta_{k}}\right) d y .
\end{aligned}
$$

Finally, using [47, Eq. (3.471.9)] for the integral in (43) yields the CDF of $Z_{k}^{(S C)}$ as in (21).

\section{The derivations for the SNR}

The message is transmitted from the sensor node $k$-th to the $j$-th antenna of the HAP can be expressed as follows

$\gamma_{k j}=\frac{P_{k} g_{k j}}{W N_{0}}=\frac{\eta_{k} t_{0 k} \gamma_{0} f_{k} g_{k j} d_{k}^{-2 \alpha}}{t_{k}}, \quad \forall j \in\{1,2, \ldots, M\}$.

Further, the HAP can process the received message from the $S_{k}$ following the SC or MRC technique. Accordingly, the received SNR at the HAP using MRC technique can be expressed as

$\gamma_{k}^{(M R C)}=\sum_{j=1}^{M} \gamma_{k j}=\frac{\eta_{k} t_{0 k} \gamma_{0} d_{k}^{-2 \alpha} f_{k}}{t_{k}} \sum_{j=1}^{M} g_{k j}$.

Setting $g_{k}^{(M R C)}=\sum_{j=1}^{M} g_{k j}$, we can rewrite (50) as

$\gamma_{k}^{(M R C)}=\frac{\eta_{k} t_{0 k} \gamma_{0} d_{k}^{-2 \alpha} f_{k} g_{k}^{(M R C)}}{t_{k}}$

If the HAP uses the SC technique, the received SNR at the HAP can be expressed as

$$
\begin{aligned}
\gamma_{k}^{(S C)} & =\max _{j \in\{1,2, \ldots, M\}}\left\{\gamma_{k j}\right\} \\
& =\frac{\eta_{k} t_{0 k} \gamma_{0} d_{k}^{-2 \alpha} f_{k}}{t_{k}} \max _{j \in\{1,2, \ldots, M\}}\left\{g_{k j}\right\} .
\end{aligned}
$$

Here we set $g_{k}^{(S C)}=\max _{j \in\{1,2, \ldots, M\}}\left\{g_{k j}\right\}$, the expression (52) can be rewritten as

$\gamma_{k}^{(S C)}=\frac{\eta_{k} t_{0 k} \gamma_{0} f_{k} g_{k}^{(S C)} d_{k}^{-2 \alpha}}{t_{k}}$ 
Finally, we obtain the expression (8) from (51) and (53).

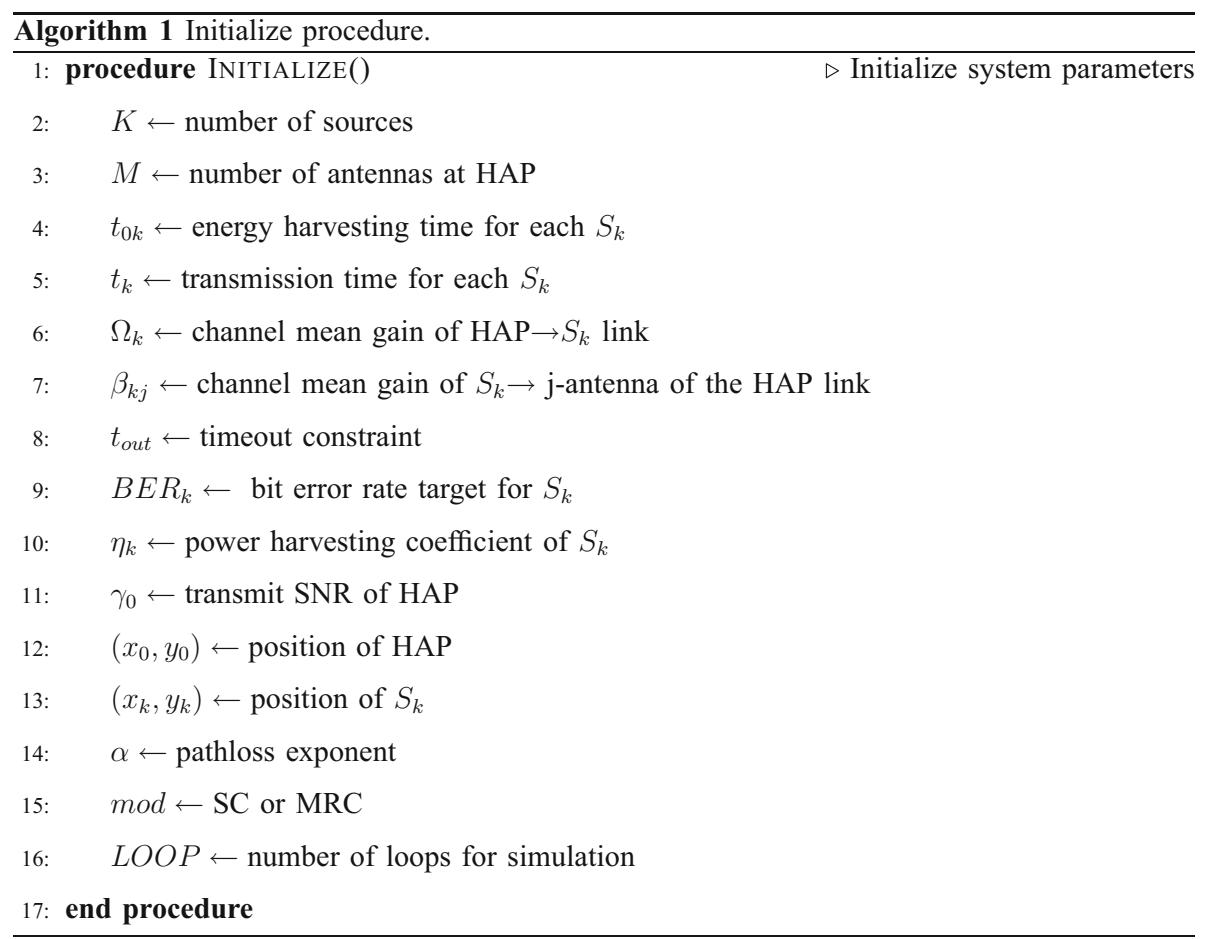




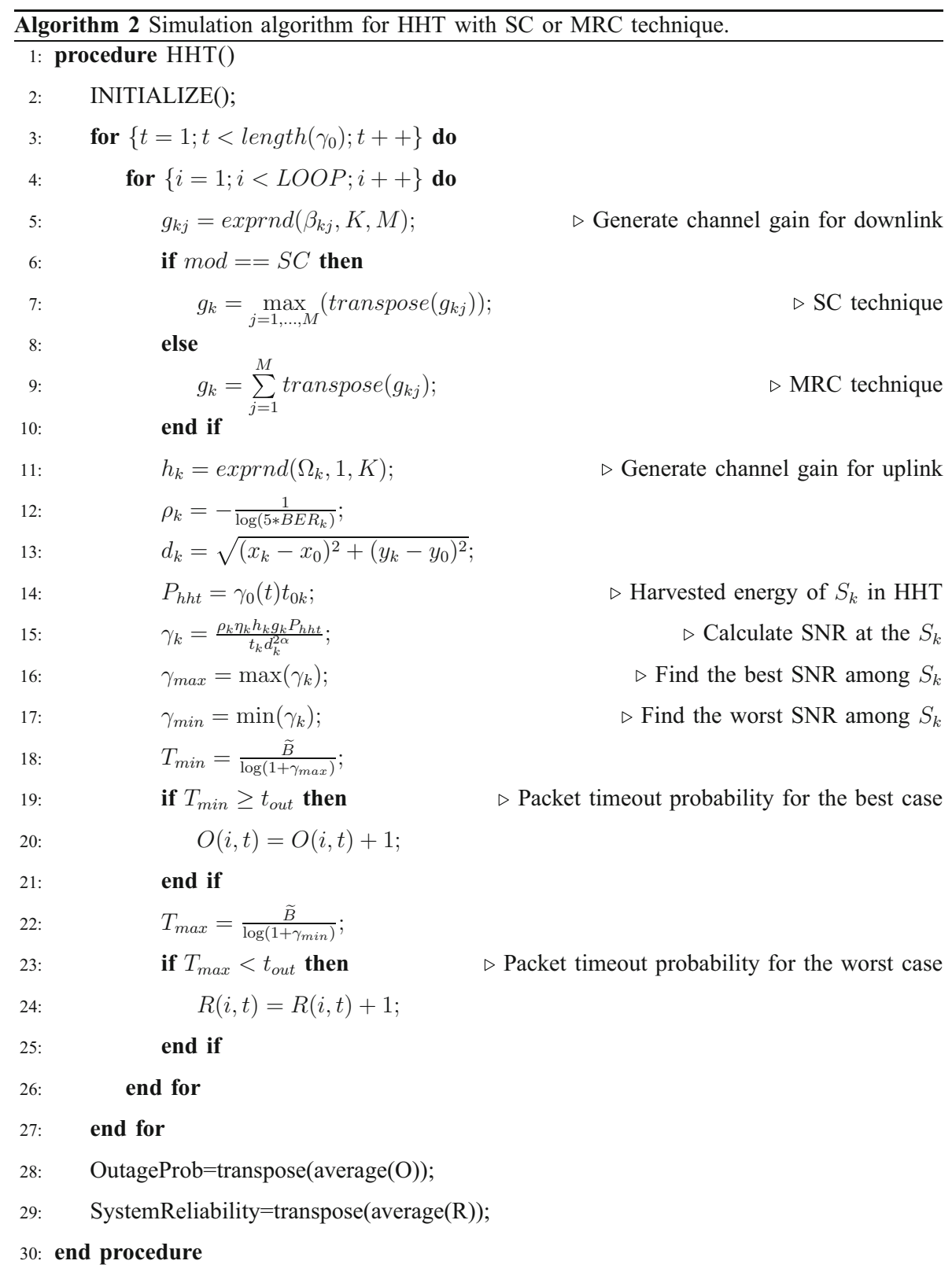

where $\operatorname{exprnd}(\cdot, \cdot, \cdot)$, transpose $(\cdot)$, and $\operatorname{average}(\cdot)$ are exponential random generating function, transpose function, and average function, respectively. 


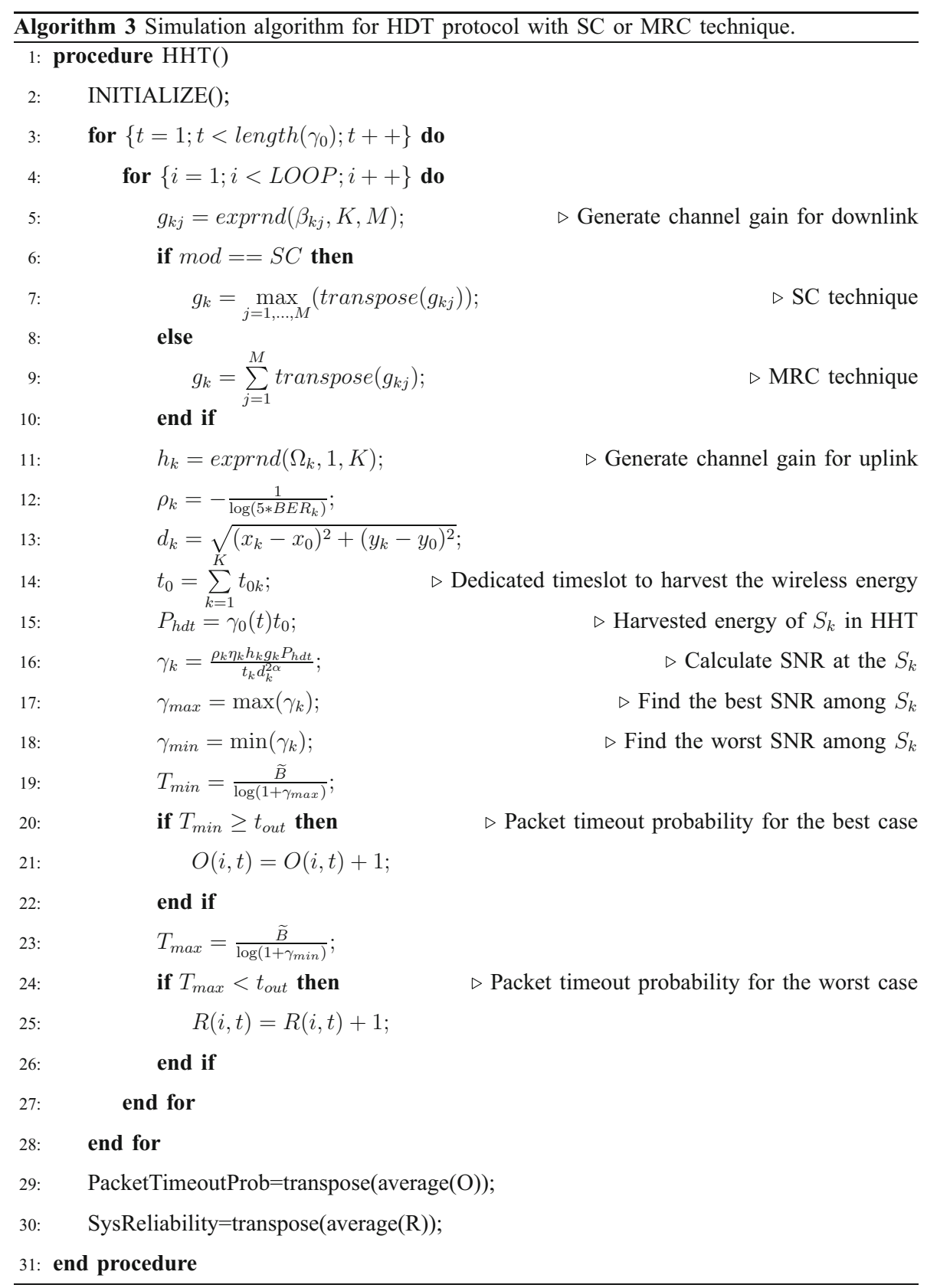

\section{References}

1. Chen, D., Nixon, M., \& Mok, A. (2010). WirelessHART(TM): Real-time mesh network for industrial automation. Berlin: Springer.

2. Nixon, M. (July 2012). A comparison of WirelessHART and ISA100.11a, in White paper.

3. Akerberg, J., Gidlund, M., \& Bjorkman, M. (July 2011). Future research challenges in wireless sensor and actuator networks targeting industrial automation. In Proceedings IEEE international conference on industrial informatics, Caparica, Lisbon (pp. 410-415).

4. Jin-Zhao, L., Xian, Z., \& Yun, L. (2009). A minimum-energy path-preserving topology control algorithm for wireless sensor networks. International Journal of Automation and Computing, 6(3), 295. doi:10.1007/s11633-009-0295-0.

5. Feng, L., Yun, L., Weiliang, Z., Qianbin, C., \& Weiwen, T. (2006). An adaptive coordinated MAC protocol based on dynamic power management for wireless sensor networks. In Proceedings international conference on wireless communications and mobile computing, New York, NY, USA (pp. 1073-1078). ACM. doi:10.1145/1143549.1143764

6. Li, Y., Yu, N., Zhang, W., Zhao, W., You, X., \& Daneshmand, M. (Apr. 2011). Enhancing the performance of LEACH protocol in wireless sensor networks. In Proceedings of IEEE conference on computer communications workshops (pp. 223-228).

7. Cao, B., Ge, Y., Kim, C. W., Feng, G., Tan, H. P., \& Li, Y. (2013). An experimental study for inter-user interference 
mitigation in wireless body sensor networks. IEEE Sensors Journal, 13(10), 3585-3595.

8. Barac, F., Caiola, S., Gidlund, M., Sisinni, E., \& Zhang, T. (2014). Channel diagnostics for wireless sensor networks in harsh industrial environments. IEEE Sensors Journal, 14(11), 3983-3995.

9. Güngör, V. Ç., \& Hancke, G. P. (2013). Industrial wireless sensor networks: Applications, protocols, and standards. CRC Press, Taylor \& Francis Group.

10. Scheible, G., Dzung, D., Endresen, J., \& Frey, J.-E. (2007). Unplugged but connected design and implementation of a truly wireless real-time sensor/actuator interface. EEE Industrial Electronics Magazine, 1(2), 25-34.

11. Shinohara, N. (2011). Power without wires. IEEE Microwave Magazine, 12(7), S64-S73.

12. Liu, V., Parks, A., Talla, V., Gollakota, S., Wetherall, D., \& Smith, J. R. (2013). Ambient backscatter: Wireless communication out of thin air. In Proceedings of the ACM conference on SIGCOMM, New York, USA (pp. 39-50). ACM.

13. Atallah, R., Khabbaz, M., \& Assi, C. (2016). Energy harvesting in vehicular networks: A contemporary survey. IEEE Wireless Communications, 23(2), 70-77.

14. He, Y., Cheng, X., Peng, W., \& Stuber, G. L. (2015). A survey of energy harvesting communications: Models and offline optimal policies. IEEE Communications Magazine, 53(6), 79-85.

15. Finkenzeller, K., Muller, D., \& Handbook, R. F. I. D. (2010). Fundamentals and applications in contactless smart cards, radio frequency identification and near-field communication (3rd ed.). Hoboken: Wiley.

16. Kang, X., Ho, C. K., \& Sun, S. (2015). Full-duplex wirelesspowered communication network with energy causality. IEEE Transactions on Wireless Communications, 14(10), 5539-5551.

17. Zhou, S., Chen, T., Chen, W., \& Niu, Z. (2015). Outage minimization for a fading wireless link with energy harvesting transmitter and receiver. IEEE Journal on Selected Areas in Communications, 33(3), 496-511.

18. Wu, T., \& Yang, H.-C. (2014). RF energy harvesting with cooperative beam selection for wireless sensors. IEEE Wireless Communications Letters, 3(6), 585-588.

19. Morsi, R., Michalopoulos, D. S., \& Schober, R. (Nov. 2014). Onoff transmission policy for wireless powered communication with energy storage. In Proceedings of conference on signals systems and computers, Pacific Grove, California, USA (pp. 1676-1682).

20. Wu, T.-Q., \& Yang, H.-C. (2015). On the performance of overlaid wireless sensor transmission with RF energy harvesting. IEEE Journal on Selected Areas in Communications, 33(8), 1693-1705.

21. Gu, Y., \& Aissa, S. (2015). RF-based energy harvesting in decode-and-forward relaying systems: Ergodic and outage capacities. IEEE Transactions on Wireless Communications, 14(11), 6425-6434.

22. Zhong, C., Chen, X., Zhang, Z., \& Karagiannidis, G. K. (2015). Wireless powered communications: Performance analysis and optimization. IEEE Transactions on Communications, PP(99), 1.

23. Chen, Y. (2015). Energy harvesting AF relaying in the presence of interference and Nakagami- $m$ fading. IEEE Transactions on Wireless Communications, $P P(99), 1$.

24. Zhu, G., Zhong, C., Suraweera, H.-A., Karagiannidis, G.-K., Zhang, Z., \& Tsiftsis, T.-A. (2015). Wireless information and power transfer in relay systems with multiple antennas and interference. IEEE Transactions on Communications, 63(4), $1400-1418$

25. Rao, S., \& Mehta, N. B. (2014). Hybrid energy harvesting wireless systems: Performance evaluation and benchmarking. IEEE Transactions on Wireless Communications, 13(9), 4782-4793.
26. Ishibashi, K., Ho, C., \& Krikidis, I. (2015). Diversity-multiplexing tradeoff of dynamic harvest-and-forward cooperation. IEEE Wireless Communications Letters, PP(99), 1.

27. Zeng, Y., \& Zhang, R. (2015). Full-duplex wireless-powered relay with self-energy recycling. IEEE Wireless Communications Letters, 4(2), 201-204.

28. Feghhi, M. M., Abbasfar, A., \& Mirmohseni, M. (2014). Performance analysis for energy harvesting communication protocols with fixed rate transmission. IET Communications, $8(18)$, 3259-3270.

29. Shi, Q., Peng, C., Xu, W., Hong, M., \& Cai, Y. (2016). Energy efficiency optimization for MISO SWIPT systems with zeroforcing beamforming. IEEE Transactions on Signal Processing, 64(4), 842-854.

30. Ahmed, I., Phan, K., \& Le-Ngoc, T. (2016). Optimal stochastic power control for energy harvesting systems with delay constraints. IEEE Journal on Selected Areas in Communications, $P P(99), 1-1$.

31. Lei, L., Kuang, Y., Shen, X. S., Yang, K., Qiao, J., \& Zhong, Z. (2016). Optimal reliability in energy harvesting industrial wireless sensor networks. IEEE Transactions on Wireless Communications, 15(8), 5399-5413.

32. Ahmed, I., Phan, K. T., \& Le-Ngoc, T. (Dec. 2015). Optimal stochastic power control for energy harvesting systems with statistical delay constraint. In Proceedings of IEEE GLOBECOM, San Diego, USA (pp. 1-6).

33. Luo, Y., Zhang, J., \& Letaief, K. B. (2016). Transmit power minimization for wireless networks with energy harvesting relays. IEEE Transactions on Communications, 64(3), 9871000.

34. Zou, Y., Li, X., \& Liang, Y. C. (2014). Secrecy outage and diversity analysis of cognitive radio systems. IEEE Journal on Selected Areas in Communications, 32(11), 2222-2236.

35. Yue, J., Ma, C., Yu, H., \& Zhou, W. (2013). Secrecy-based access control for device-to-device communication underlaying cellular networks. IEEE Communications Letters, 17(11), $2068-2071$.

36. Suraweera, H. A., Smith, P. J., \& Shafi, M. (2010). Capacity limits and performance analysis of cognitive radio with imperfect channel knowledge. IEEE Transactions on Vehicular Technology, 59(4), 1811-1822.

37. Tran, H., Kaddoum, G., Gagnon, F., \& Sibomana, L. (2017). Cognitive radio network with secrecy and interference constraints. Physical Communication, 22, 32-41.

38. Lu, X., Wang, P., Niyato, D., Kim, D. I., \& Han, Z. (2015). Wireless networks with RF energy harvesting: A contemporary survey. EEE Communications Surveys \& Tutorials, 17(2), 757-789.

39. Shinohara, N. (2013). Wireless power transfer via radiowaves. Hoboken: Wiley.

40. Khan, F. A., Tourki, K., Alouini, M. S., \& Qaraqe, K. A. (Oct. 2012). Delay analysis of a point-to-multipoint spectrum sharing network with CSI based power allocation. In Proceedings of IEEE international symposium on dynamic spectrum access networks, Bellevue, WA, USA (pp. 235-241).

41. Khan, F. A., Tourki, K., Alouini, M. S., \& Qaraqe, K. A. (2014). Delay performance of a broadcast spectrum sharing network in Nakagami-m fading. IEEE Transactions on Vehicular Technology, 63(3), 1350-1364.

42. Tran, H., Duong, T., \& Zepernick, H.-J. (February 2011). Queuing analysis for cognitive radio networks under peak interference power constraint. In Proceedings of ieee international symposium on wireless and pervasive computing Mondena, Italy (pp. 1-5).

43. Tran, H., Zepernick, H.-J., Phan, H., \& Sibomana, L. (2015). Performance analysis of a cognitive radio network with a 
buffered relay. IEEE Transactions on Vehicular Technology, 64(2), 566-579.

44. Mehta, N., Sharma, V., \& Bansal, G. (2011). Performance analysis of a cooperative system with rateless codes and buffered relays. IEEE Transactions on Wireless Communications, 10(4), 1069-1081.

45. Qiu, X., \& Chawla, K. (1999). On the performance of adaptive modulation in cellular systems. IEEE Transactions on Communications, 47(6), 884-895.

46. Liu, E., Zhang, Q., \& Leung, K. (2011). Asymptotic analysis of proportionally fair scheduling in Rayleigh fading. IEEE Transactions on Wireless Communications, 10(6), 1764-1775.

47. Gradshteyn, I., \& Ryzhik, I. (2015). Table of integrals, series, and products, eight (8th ed.). Amsterdam: Elsevier.

48. Yu, K., Gidlund, M., Åkerberg, J., \& Björkman, M. (Sep. 2011). Reliable and low latency transmission in industrial wireless sensor networks. In Proceedings on first international workshop on wireless networked control systems, Niagara Falls, ON, Canada.

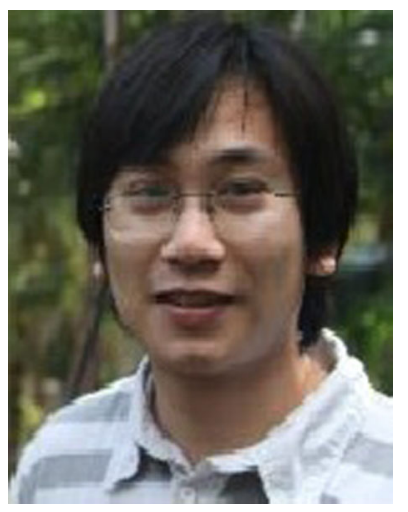

Hung Tran was born in Hanoi, Vietnam, in 1980. He received the B.S. degree and M.S. degree in information technology from Vietnam National University, Hanoi, in 2002 and 2006, respectively, and the Ph.D. degree from the School of Computing, Blekinge Institute of Technology, Karlskrona, Sweden, in 2013. In 2014, he joined the Electrical Engineering Department, École de Technologie Supérieure, Montreal, Canada. He is currently a Post-Doctoral Researcher with Mälardalen University, Sweden. His research interests include cognitive radio networks, cooperative communication systems, millimeter wave communications, energy harvesting and security communications at physical layer.

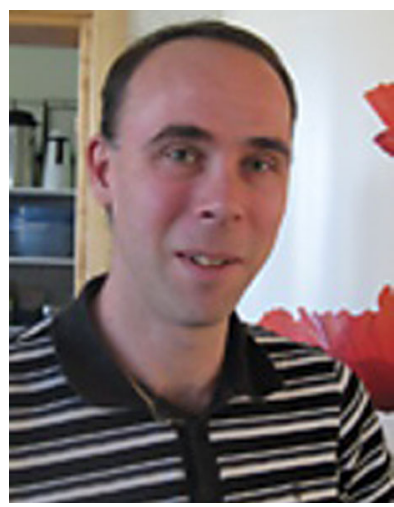

Johan Åkerberg is an adjunct professor at Märlardalen University and a principal scientist at $\mathrm{ABB}$ Corporate Research in Sweden. Johan Åkerberg received his MSc and Ph.D. degree in Computer Science and Engineering from Mälardalen University, Sweden. $\mathrm{He}$ is also an active IEEE senior member organizing special sessions, holding tutorials and acting as TCP member in various distinguished IES conferences. $\mathrm{He}$ is mainly working with communication for embedded real-time systems in industrial

automation and is frequently invited to give talks to governmental bodies, international universities and automation fairs. He has close to 20 years experience within $A B B$ in various positions such as $R \& D$ project manager, industrial communication specialist and product manager.

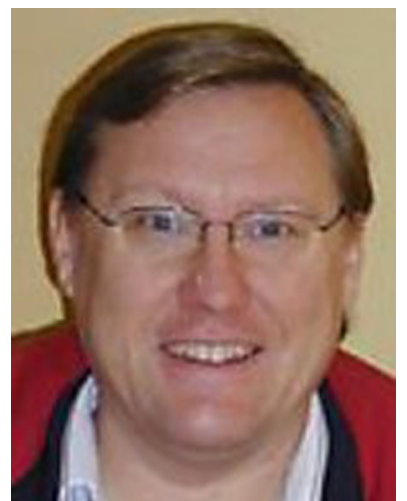

Mats Björkman is Professor in Computer Communication at Mälardalen University since 2001. He received his MSc in Computer Science in 1986 from Uppsala University, and his Ph.D. in Computer Systems in 1993 also from Uppsala University. Previously, Mats has worked as a researcher at the Swedish Institute of Computer Science (SICS) in Stockholm, Sweden, and at the University of Arizona in Tucson, Arizona. His current research interests include computer network performance and predictability issues, and communication for embedded systems, sometimes called CyberPhysical Systems (CPS) or Internet of Things (IoT). One current focus is methods and methodology for delay prediction, another current focus is reliability issues (safety, security) for wireless networks. Application areas include industrial automation and health applications.

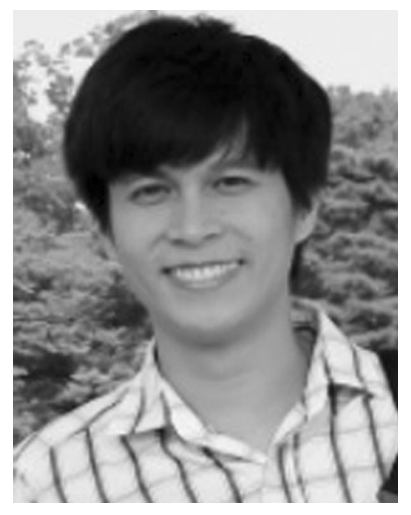

and heterogeneous networks.
Ha-Vu Tran received the bachelor's degree in Electronic and Telecommunication Engineering from Hue University of Sciences, Vietnam, in 2012, the master's degree in Electronics and Radio Engineering from Kyung Hee University, South Korea, in 2015. He is currently pursuing the $\mathrm{Ph} . \mathrm{D}$. degree with the École de Technologie Supérieure, University of Québec, Canada. His research interests include wireless power transfer, green communications, 